\title{
Research Summary of Kishi Group
}

\author{
Yoshito Kishi
}

Our research is centered on the chemistry of natural products with high biological activity, ranging from structure elucidation to total synthesis. For the simplicity of presentation, the research activities carried out in our laboratory over the past three and a half decades are classified into the following ten subgroups in this summary. The inspiration and motivation for these studies have often originated from our curiosities on certain natural products. In this context, palytoxin has served as the major source of our inspiration for the past two and a half decades: see, Y. Kishi, "Palytoxin: An Inexhaustible Source Of Inspiration-Personal Perspective-", Tetrahedron, 58, 6239 (2002).

\section{Isolation and Structure Elucidation of Natural Products}

Under the supervision of the late Professors Yoshimasa Hirata and Toshio Goto, I received the graduate education at Nagoya University. My graduate work was concerned with the isolation/structure elucidation of puffer fish poison tetrodotoxin (1) and Cypridina luciferin (2), the enzyme substrate for the Cypridina bioluminescent system.

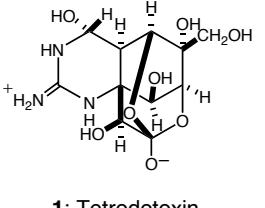

1: Tetrodotoxin

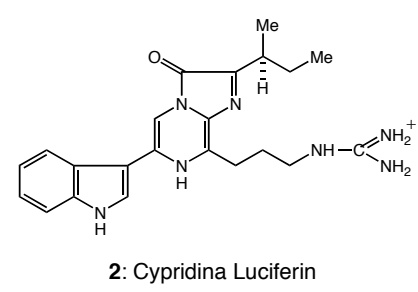

2: Cypridina Luciferin

\section{Structure Elucidation via Organic Synthesis}

The first example in this area was the elucidation of the geometric stereochemistry of Latia luciferin (3), the enzyme substrate for the Latia bioluminescent system, by organic synthesis. $E$ - and $Z$-enol formates were stereospecifically synthesized from the corresponding $E$ - and $Z$ - $\alpha, \beta$-unsaturated aldehydes, respectively. Their ${ }^{1} \mathrm{H}$ NMR spectra were found surprisingly similar. This result was instructive in many respects and, through this work, it was recognized that contemporary synthetic organic chemistry offers unlimited opportunities to address structural problems in a unique way.

Photoprotein aequorin, krill and dinoflagellate luciferins (4 and 5), cytoblastin (6), tolyporphin A (7), mycolactones (8a-c), and pteriatoxins (24a-c) are classified in this sub-group, where organic synthesis played the key role in structure elucidation. Among them, the work on the functional moiety of photoprotein aequorin deserves a comment; specifically ${ }^{13} \mathrm{C}$-enriched coelenterazine (9), the light-emitting chromophore of photoprotein aequorin, was used to suggest that $\mathbf{9}$ is incorporated as the peroxide $\mathbf{1 0}$ in the photoprotein. 

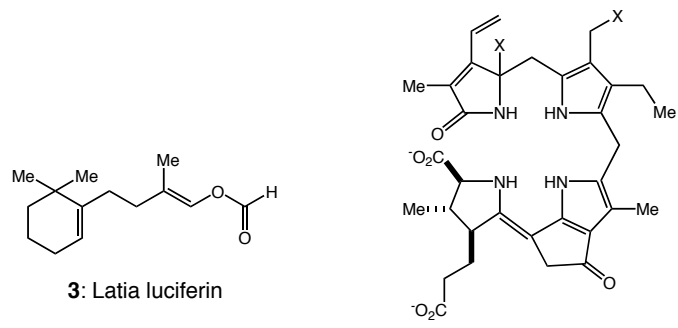

4: Krill luciferin $(\mathrm{X}=\mathrm{OH})$

5: Dinoflagellate luciferin $(X=H)$
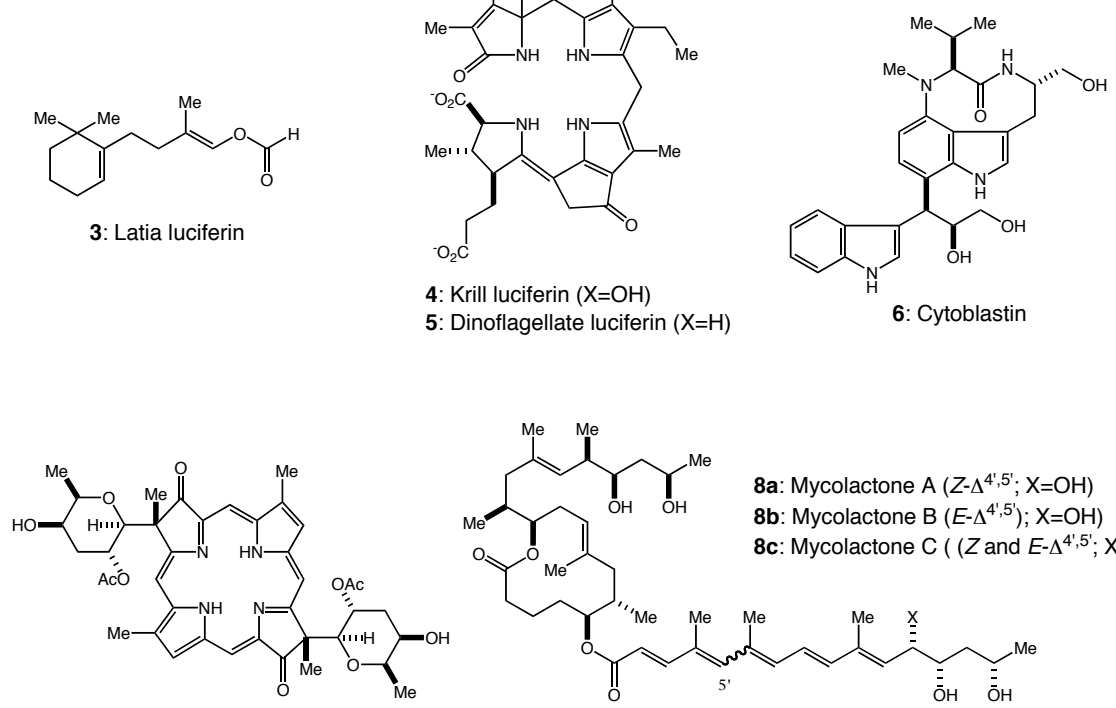

7: Tolyporphin A

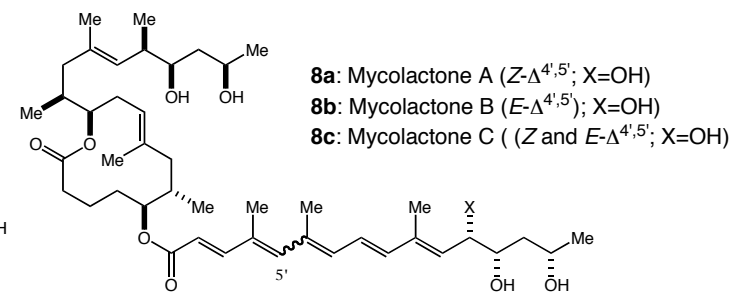

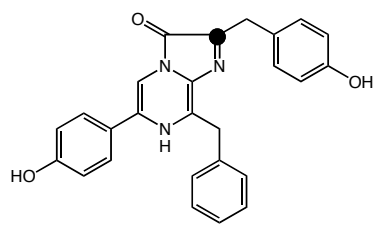

9: Coelenterazine

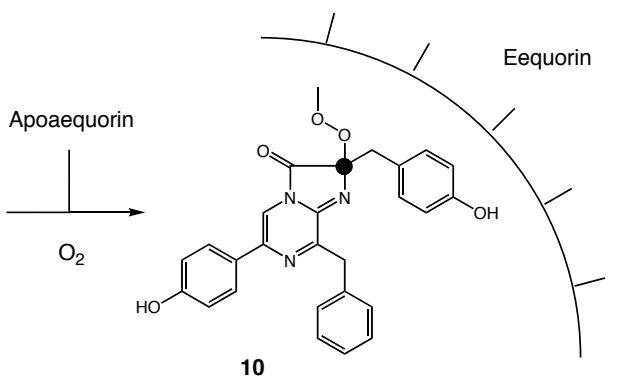

\section{Total Synthesis of Natural Products with Highly Condensed Functional Groups}

One of our major efforts has been directed toward a total synthesis of natural products with highly condensed functional groups. The work on the following natural products is classified in this sub-group: echinulin (11), tetrodotoxin (1), saxitoxin (12), gliotoxins (13), sporidesmins A and B (14a and $\mathbf{b})$, mitomycins A and C (15a and $\mathbf{b})$, histrionicotoxins (16), austamides (17), gephyrotoxins (18), aklavinone/11deoxydaunomycinone (19/20), myclamides/onnamide A (21/22), cytoblastin (6), tolyporphin A (7), pinnatoxins/pteriatoxins (23/24), and batrachotoxinin A/batrachotoxin $(25 \mathrm{a} / \mathrm{b})$. 

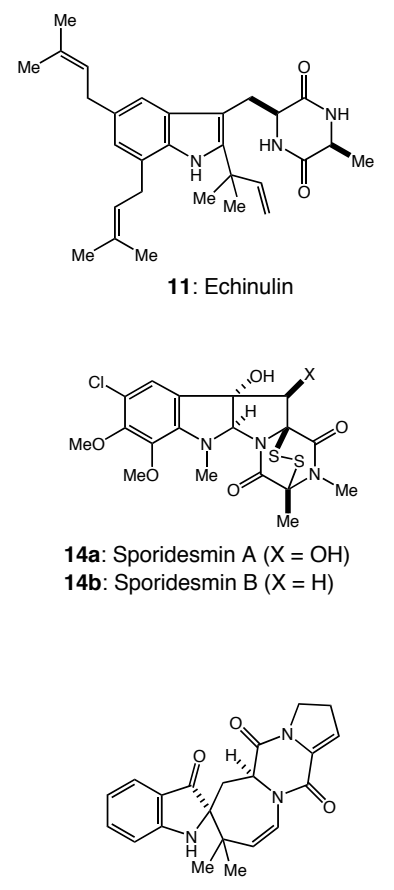

17: Austamide

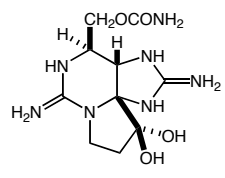

12: Saxitoxin

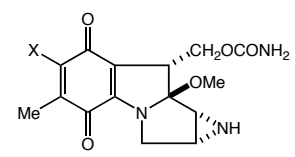

15a: Mitomycin $\mathrm{A}(\mathrm{X}=\mathrm{OMe})$ 15b: Mitomycin $\mathrm{C}\left(\mathrm{X}=\mathrm{NH}_{2}\right)$

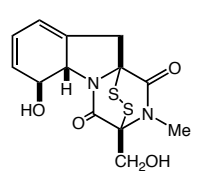

13: Gliotoxin

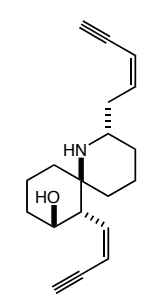

16: Histrionicotoxin

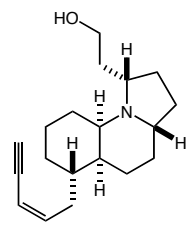

18: Gephyrotoxin

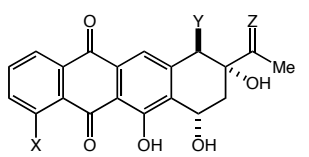

19: Aklavinone $\left(X=\mathrm{OH} ; \mathrm{Y}=\mathrm{CO}_{2} \mathrm{Me} ; \mathrm{Z}=\mathrm{H}_{2}\right)$

20: 11 -Deoxydaunomycinone
$(\mathrm{X}=\mathrm{OMe} ; \mathrm{Y}=\mathrm{H} ; \mathrm{Z}=\mathrm{O})$

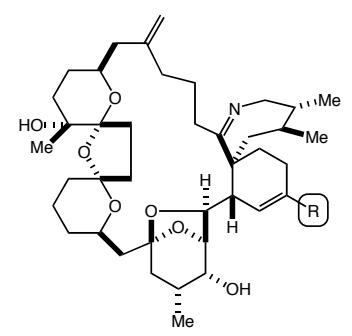

Mycalamide $A(R=H ; x$ 21b: Mycalamide $B(R=M e ; X=O H)$ 22: Onnamide $A(R=H$;<smiles>[Y]=CCC/C=C\C=C\C=C\C(=O)NC(CCCNC(N)=[NH2+])C(=O)[O-]</smiles>

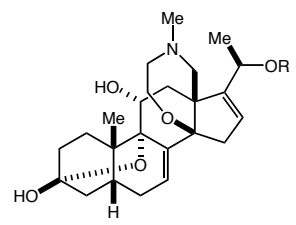

25a: Batrachotoxinin $A(R=H)$ 25b: Batrachotoxin $\mathrm{R}$

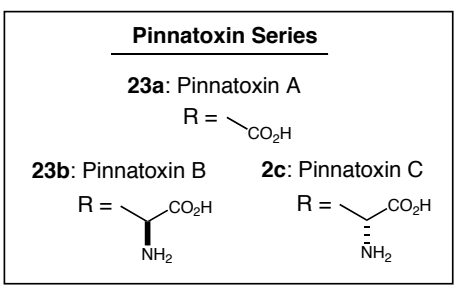

Pteriatoxin Series

24a: Pteriatoxin A

$$
\mathrm{R}=\overbrace{\mathrm{OH}} \overbrace{\mathrm{NH}_{2}}^{\mathrm{CO}_{2} \mathrm{H}}
$$

24b: Pteriatoxin $B \quad$ 24c: Pteriatoxin $C$

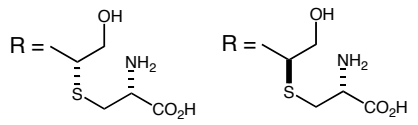

\section{Acyclic Stereocontrol}

This program was undertaken in 1974, when our group moved from Nagoya University to Harvard. Our motivation arose from the question around the strategy and 
tactics for a synthesis of the polyether class of antibiotics such as lasalocid A (26) and monensin (27). In a broad sense, our interests were, and still are, centered in advancing a general (empirical) rule to predict the stereochemical course for a given conformationally flexible acyclic system. We were aware of the difficulties encountered in this approach, but we could not deny the temptation of testing its feasibility and practicability for its enormous potential.

After a considerable induction period, this program flourished, resulting in a total synthesis of lasalocids (26), monensins (27), rifamycins (28), calcimycin (A23187) (29), naracins/salinomycins (30/31), palytoxin carboxylic acid/palytoxin (32/33), and altohyrtin A (spongistatin 1) (34).

Importantly, through these syntheses, our group uncovered a number of highly interesting and exciting theoretical points and advanced the rules to predict the major product for a given conformationally flexible acyclic system. 


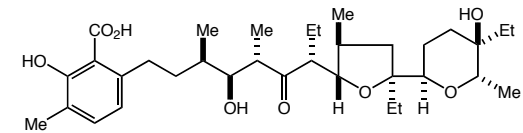

26: Lasalocid A

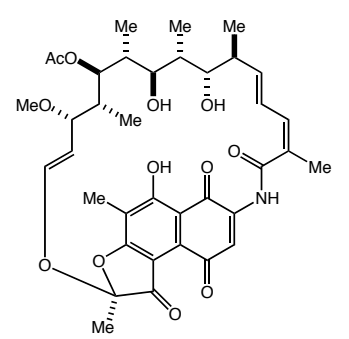

28: Rifamycin S
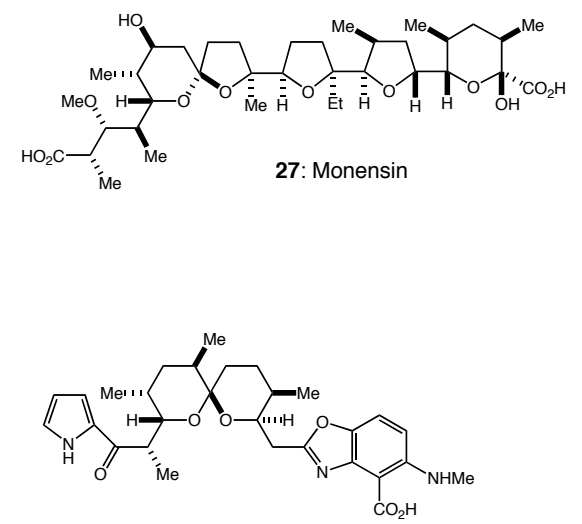

29: Calcimycin (A23187)

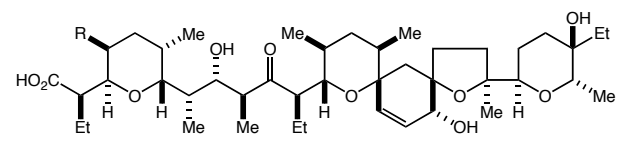

30: $\operatorname{Narasin}(\mathrm{R}=\mathrm{Me})$

31: Salinomycin $(\mathrm{R}=\mathrm{H})$

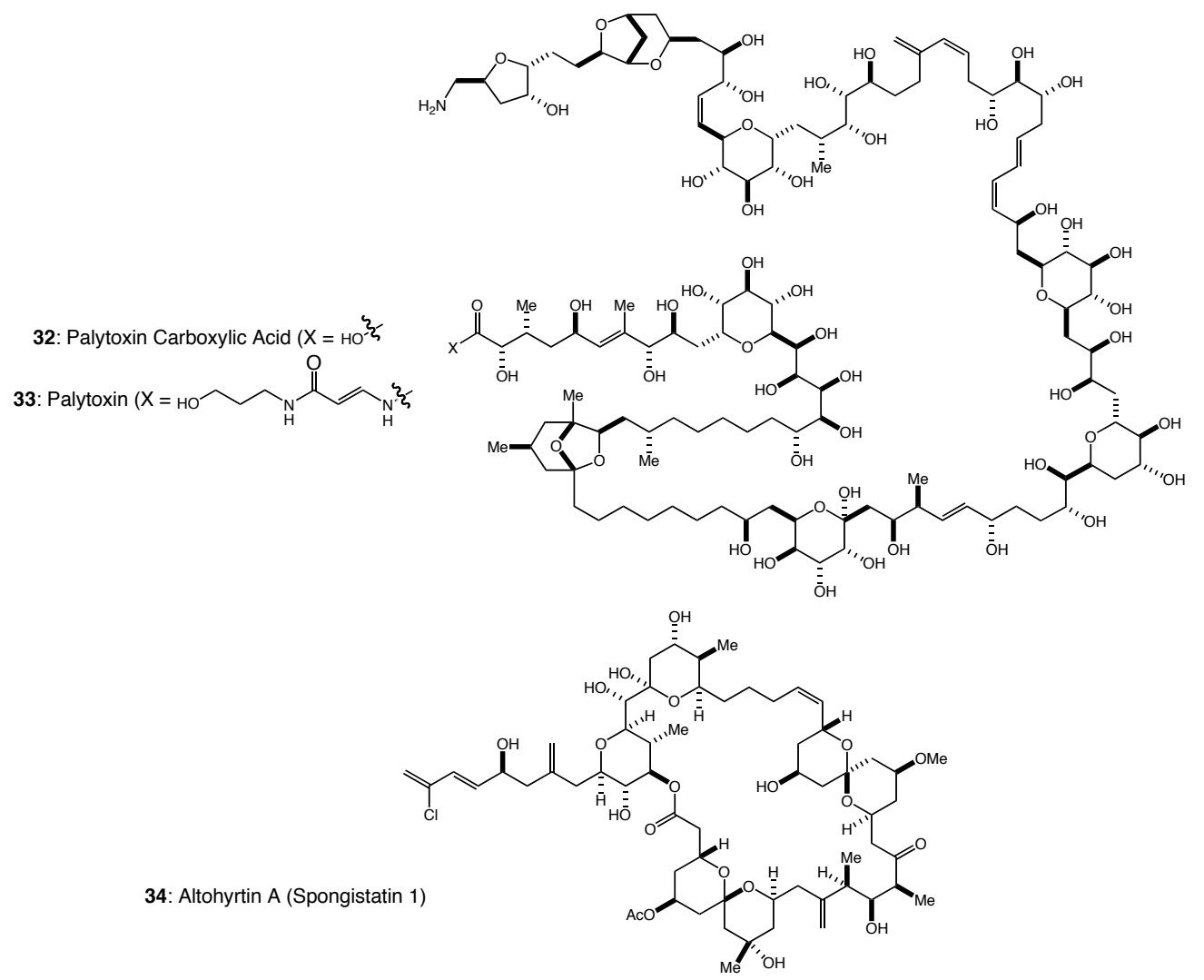

\section{New Synthetic Strategy and Synthetic Reactions}

Our group was engaged with total syntheses of various natural products. In order to carry out these syntheses, not only new synthetic strategies but also new synthetic 
methods were extensively investigated. In other words, we took on natural product syntheses, when some opportunities were seen to develop new synthetic strategies and reactions. For example, the syntheses of the gliotoxin/sporidesmin-class of natural products required the development of a synthetic route to epidithiodiketopiperazines, since there was virtually no synthetic method known to construct them.

These new synthetic strategies and/or new synthetic reactions were reported along with the total syntheses. Among them, however, $\mathrm{Ni}$ (II)/Cr(II)-mediated coupling reaction deserves further comments. This reaction was discovered in conjunction with the synthesis of $\mathrm{C} 8-\mathrm{C} 22$ segment of palytoxin. The $\mathrm{Ni}(\mathrm{II}) / \mathrm{Cr}(\mathrm{II})$-mediated coupling allows a carbon-carbon bond formation between alkenyl halides and aldehydes. While traditional organometallic reagents can usually achieve this transformation, there are several unique characteristics of this reaction. In our view, this coupling reaction demonstrates its uniqueness most when applied to polyfunctional substrates for which conventional organometallic reagents are difficult to apply. To study further its scope and limitations, we had purposely chosen to use the $\mathrm{Ni}(\mathrm{II}) / \mathrm{Cr}(\mathrm{II})$-mediated coupling reaction as the key bond-forming step for the synthesis of various natural and non-natural products, including ophiobolin C (35), taxicins/taxol (36/37: Harvard Dissertaion by Michael $\mathrm{H}$. Kress (1995), Christopher Sheng (1998), and Sungtaek Lim (2000)), halichondrins (38), and others.
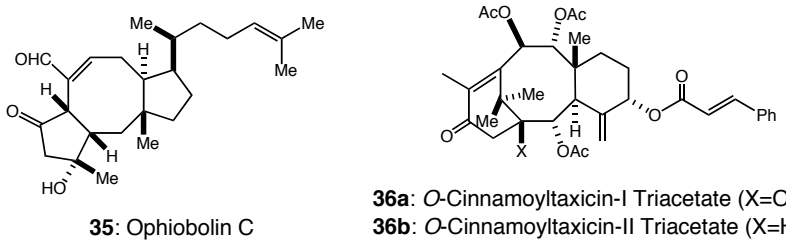

36a: O-Cinnamoyltaxicin-I Triacetate $(X=\mathrm{OH})$ 36b: O-Cinnamoyltaxicin-II Triacetate $(\mathrm{X}=\mathrm{H})$
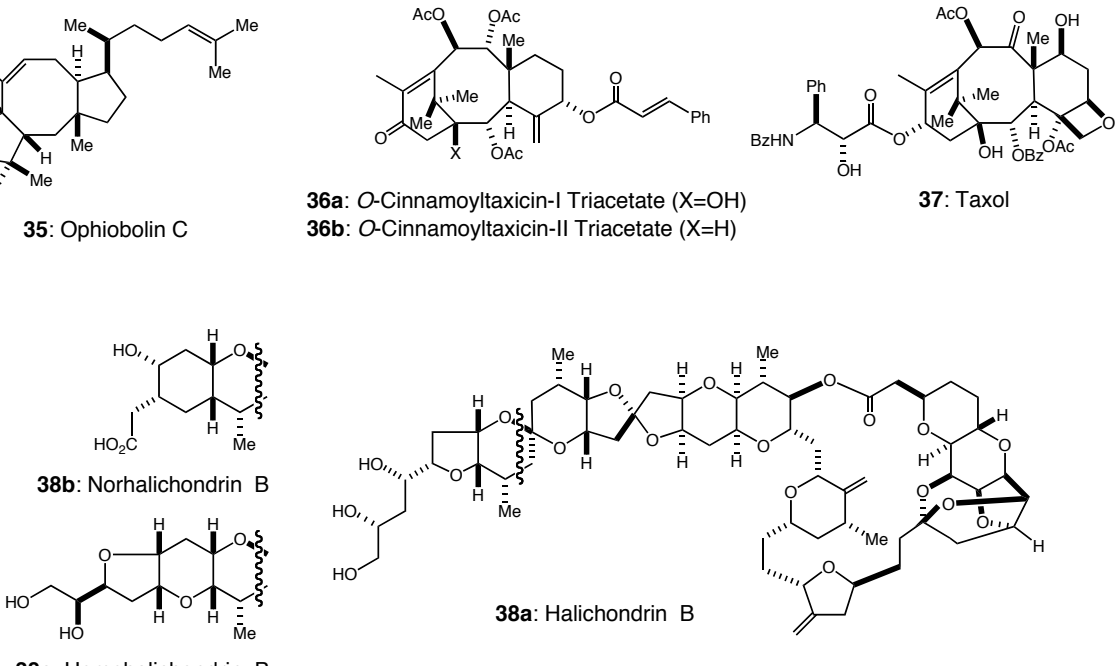

38c: Homohalichondrin B

Among them, the synthesis of halichondrins (38), a class of polyether macrolides isolated from the marine sponge Halichondria okadai, deserves special comment. Halichondrins exhibit extraordinary in-vitro and in-vivo antitumor activity. However, the very limited supply of halichondrins from natural sources prevented further evaluation of their potential clinical application. Coupled with this fact, their intriguing and challenging structural features encouraged us to undertake a synthesis project on this class of natural products. In practice, we planned, and successfully executed, the assembly of halichondrin B using five $\mathrm{Ni}(\mathrm{II}) / \mathrm{Cr}$ (II)-mediated coupling reactions.

Perhaps the most beneficial discovery on the biological activity of halichondrin B was made by chance. Upon the completion of synthesis, the synthetic halichondrin B as well as several synthetic intermediates were sent to the National Cancer Institute and 
Eisai Research Institute (ERI) for testing the in vitro and in vivo antitumor activities. The results were sensational: the antitumor activity of halichondrin B resides in the right portion of the molecule. Based on this discovery, a patent was applied for by, and granted to, Harvard. Then, ERI reached an agreement on use of this patent with Harvard and undertook massive drug discovery efforts, through which two exceptional drug candidates have emerged. Recently, the National Cancer Institute completed the Phase I study on one of ERI's two drug candidates, thereby showing the extraordinary level of antitumor activity in man.

It should be pointed out the fact that the structural complexity of the right-half of halichondrin B, or of ERI's analogs, exceeds by far the structural complexity found in drugs on the market and/or drug candidates under development. Thus, development of an economically feasible synthesis of the right-half of halichondrin B and/or ERI's drug candidate is the key for success of this program. It is our belief that contemporary synthetic organic chemistry has the capacity and potential to meet this type of challenge. We even take this as testimony to the power of contemporary synthetic organic chemistry.

With this analysis, the research on the $\mathrm{Ni}(\mathrm{II}) / \mathrm{Cr}(\mathrm{II})$-mediated and related coupling reactions has been vigorously pursued, thereby expanding dramatically the scope of $\mathrm{Ni}(\mathrm{II}) / \mathrm{Cr}(\mathrm{II})$-mediated coupling reactions. It is now possible to perform alkenylation, alkynylation, 2-haloallylation, allylation, propargylation, alkylation, and arylation in a catalytic, asymmetric manner at the synthetically useful level. This development has had an immediate impact on various ongoing synthetic programs including halichondrins. For example, the C14-C26 building block of halichondrins can be assembled in a surprisingly concise and effective manner via successive use of $\mathrm{Co} / \mathrm{Cr}$-mediated 2-haloallylation, $\mathrm{Ni} / \mathrm{Cr}$-mediated alkenylation, and then $\mathrm{Co} / \mathrm{Cr}$-mediated alkylation.

\section{Universal NMR Database}

The program "structure elucidation via organic synthesis" gradually evolved from the approach adopted for palytoxin, to the method tested in AAL toxins/fumonisins $(\mathbf{3 9 a} / \mathbf{b})$ and also maitotoxin (40), and finally to the concept of the universal NMR database approach. With use of small stereoclusters such as contiguous dipropionates, 1,3,5-triols, contiguous polyols and others, the logic and concept of the universal NMR database approach was successfully developed. In order to demonstrate the reliability, usefulness, and versatility of this approach, several natural products were used, including oasomycins (41), tetrafibricin (42), and others. 

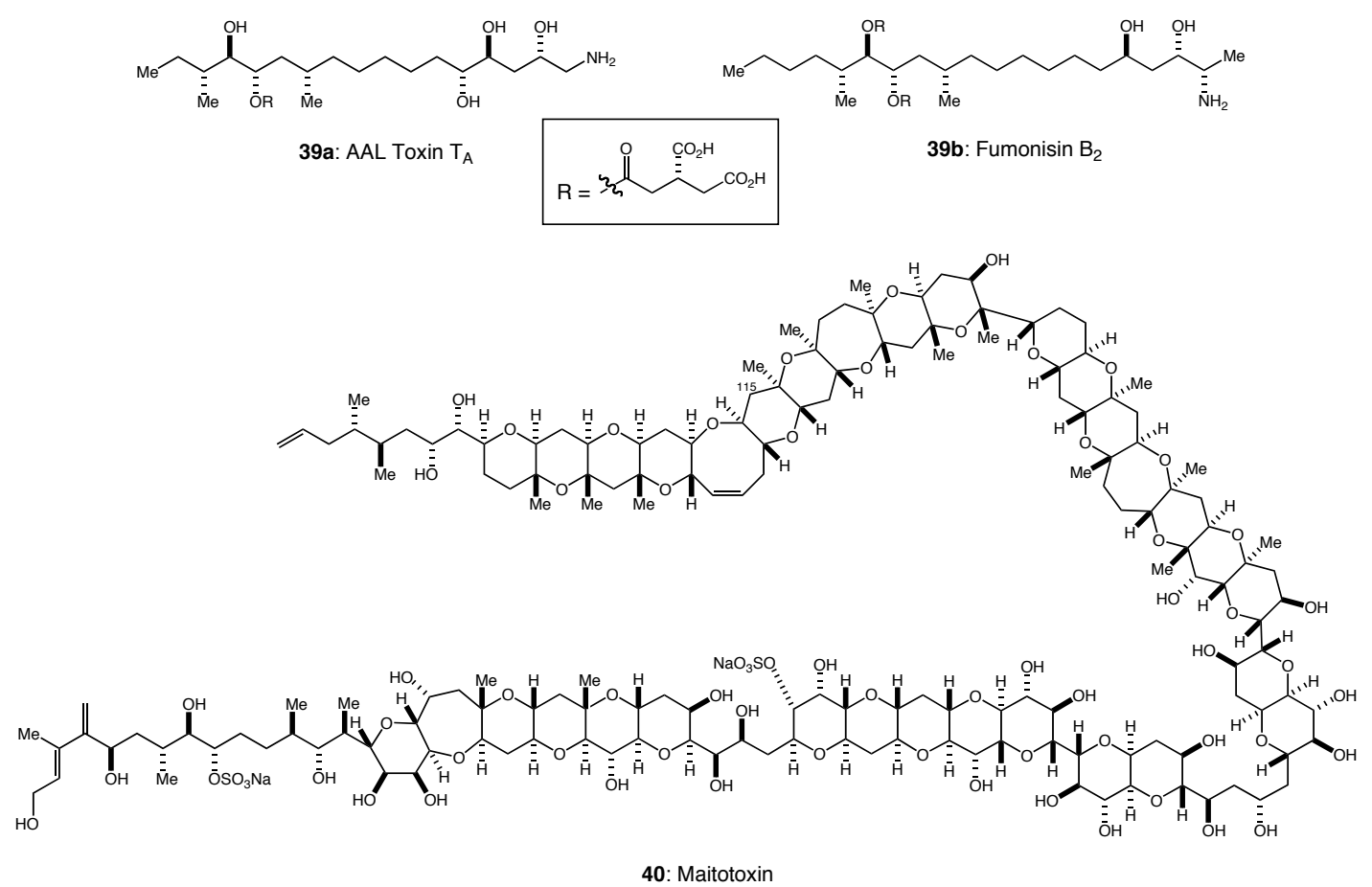

In our view, the basic logics and concepts used in this approach have matured up to the level where the relative and absolute configuration of an unknown compound can be determined without degradation and/or derivatization work. However, further development was required to deal with a specific problem such as the one found for the case of glisoprenin A (43).

The universal NMR database approach consists of the following steps: (1) to identify a stereocluster present in a given molecule, (2) to compare its NMR profile with each of the NMR profiles of all the possible diastereomers for the stereocluster, and (3) to predict its stereochemistry based on the profile-fitness. Among these steps, step 2, which involves "synthesis of all the diastereomers possible for a given sterocluster" to assemble an NMR database, requires the most significant laboratory effort and, consequently, it would be beneficial to explore the possibility of eliminating this step. In conjunction with the stereochemistry assignment of sagittamide A (44), such an approach was recently developed. 


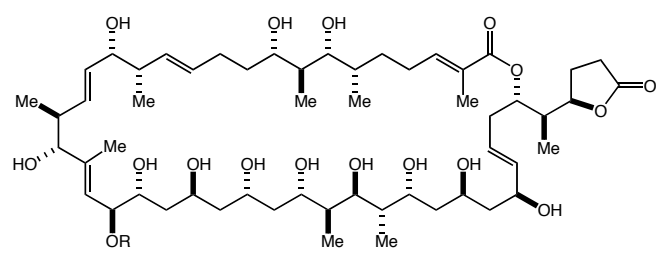

41a: Oasomycin $A: R=H$ 41b: Oasomycin $B: R=\alpha$-D-mannosyl
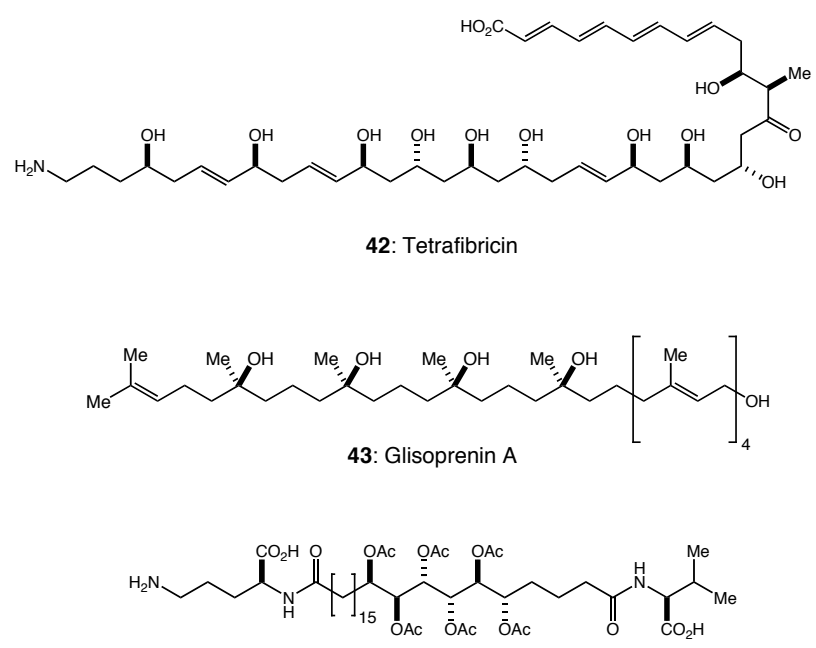

44: Sagittamide A

\section{Conformational Studies $\boldsymbol{C}$-Glycosides and Palytoxin}

As major parts of the palytoxin structure could be viewed as $C$-oligosaccharides, we became interested in comparing the conformational characteristics of $C$-glycosides with those of the corresponding $O$-glycosides. Our major goal was not to define the precise conformation for a given $C$-glycoside, but rather to introduce a useful analytical device: (1) to identify strategic steric interactions that have a significant influence on local conformational preferences and (2) to suggest rational structural modifications for modulating its global conformational profile. In order to assess local steric interactions, diamond-lattice assisted conformational analysis was introduced. With this method, 1,3diaxial-like steric interactions can be conveniently evaluated, thereby providing with a mechanism for identifying functional groups in conformationally strategic positions and programming oligosaccharide structures with modified secondary structures. Importantly, the lessons learned from the conformational analysis of structurally modified $C$-glycosides could be translated directly into the rational design of conformationally modified $O$-glycosides.

The next phase of this program was to study the carbon analog of a biologically significant substrate. Two examples, $C$-lactose (45)/lectin interaction and human Type II $\mathrm{O}(\mathrm{H})$ blood group determinant $C$-trisaccharide (46), were selected to address three issues: (1) to show that the conformational properties of this compound can be predicted and that the prediction can be experimentally tested, (2) to demonstrate that the compound can be induced to adopt different yet predictable and well-defined conformations as a result of specific, rationally designed structural modifications, and (3) to examine their effect(s) on 
the biological behaviors in comparison to the corresponding parent $O$-glycosides. Overall, the anticipated behaviors were observed in the both cases.
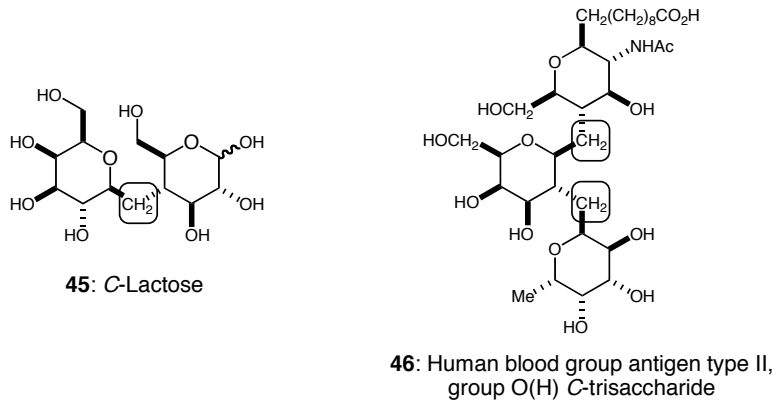

The knowledge gained through the conformational studies on $C$-glycosides allowed us to predict, and experimentally prove, the preferred conformation for the strategically chosen small segments of palytoxin. Combining the conformational preferences of these small segments yielded the preferred global conformation of palytoxin itself. In this preferred global conformation, the distance between the $C$ - and $N$-terminals was estimated to be $31 \AA$. In order to provide experimental support for the predicted global conformation, a chemical ruler based on conformationally well-defined $3_{10}$-helical oligopeptides was developed. In reference to this chemical ruler, the distance between the $C$ - and $N$-terminals of palytoxin was estimated to be $30 \AA$ via fluorescence energy transfer experiments.

\section{Synthetic 3-O-Methylmannose-containing Polysaccharides ( $\mathrm{OMMP}$ ) and 6-O- Methylglucose-containing Polysaccharides (sMGP)}

The conformational studies on $C$ - and $O$-glycosides led us to undertake a new program, 3-O-methyl-D-mannose-containing polysaccharides (MMP) and 6-O-methyl-Dglucose-containing (lipo)polysaccharides (MG(L)P), isolated from Mycobacterium smegmatis. Both MMP and $\mathrm{MG}(\mathrm{L}) \mathrm{P}$ are known to affect profoundly the fatty acid biosynthesis in Mycobacterium smegmatis; they increase the overall rate of fatty acid biosynthesis, change the product distribution, and prevent product-inhibition of the fatty acid synthetase. Both MMP and MGP were shown to exhibit interesting host/guest chemistry with C16- or longer acyl-CoA in water. These extraordinary chemical and biochemical properties warrant further investigations on the MMP/MG(L)P-classes of naturally occurring polysaccharides. Unfortunately, however, the polysaccharides from natural sources are a complex mixture of structurally closely related polysaccharides. To overcome this difficulty, synthetic 3-O-methylmannose-containing polysaccharides ( $s \mathrm{MMP}$ ) and 6-O-methyl-D-glucose-containing polysaccharides ( $s \mathrm{MGP}$ ) were designed for two major reasons: (1) synthetic polysaccharides should be available as the structurally well-defined and homogeneous materials and (2) synthetic polysaccharides should be structurally tunable for the need of investigation. Efficient and convergent synthetic routes were developed to both $s$ MMP and $s$ MGP, thereby having provided a series of $s$ MMPs $(47 ; \mathrm{n}=6$ up to $\mathrm{n}=20)$ and $s$ MGPs $(48 ; \mathrm{n}=6$ up to $\mathrm{n}=20)$. The first generation of sMMP- and sMGP-syntheses have recently been submitted for publication. 


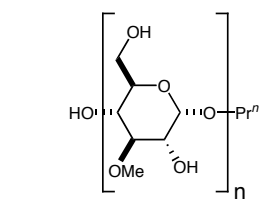

47: $s M M P$

$(\mathrm{n}=6,8,10,12,14,16,18,20)$

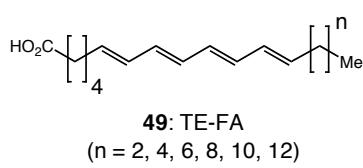

In order to demonstrate that $s$ MMP and $s$ MGP mimic natural MMP and MGP, it was necessary to measure their binding affinities with fatty acids and fatty acid-CoAs. Unfortunately, there was no reliable method available to estimate such binding affinities. For this very reason, a new project was undertaken to learn the synthesis and properties of tetraenoic fatty acids (TE-FAs) (49), thereby shedding light on intriguing aggregationbehaviors of fatty acids in aqueous solution. Some of these discoveries have recently been submitted for publication.

Besides the surprising observations on the aggregation of fatty acids, the studies on tetraenoic acids allowed us to develop a new, reliable method to estimate the binding affinities between $s$ MMPs/sMGPs and fatty acids/fatty acid-CoAs, thereby demonstrating that $s \mathrm{MMPs} / s$ MGPs exhibit the host/guest chemistry exactly as natural MMP/MGP. Excitingly, $s$ MMPs and $s$ MGPs were found to mimic beautifully the biological roles of natural MMP and MGP. Importantly, with a series of $s$ MMPs ( $n=6$ up to $n=20$ ) and $s$ MGPs ( $\mathrm{n}=6$ up to $\mathrm{n}=20$ ) and their binding affinities with fatty acids and fatty acid-CoAs in hand, it was possible to conduct the critical experiments and shed light on Nature's trick to shift the fatty acid distribution from C24-fatty acid to C16. It is an amazingly simple, even primitive, but effective trick. These results will be reported in due course.

\section{Development of Research Tools in Biology and Related Areas}

We have used organic synthesis as the key tool to address the question of biological importance. The first example in this area was the aplysiatoxin synthesis, in conjunction with the three-dimensional model-building exercise on the pharmacophore of protein kinase $\mathrm{C}(\mathrm{PKC})$. $\mathrm{PKC}$ is a serine/threonine kinase known to be involved in diverse signal transduction processes. The enzyme is normally quiescent, but activated by the endogenous activator $(S)$-diglycerides (DAGs). PKC is activated not only by the endogenous DAGs, but also by the structurally diverse tumor promoters. The marine natural products aplysiatoxins are known to be the effective activators of PKC. The research plan consisted of two phases: (1) to establish a synthetic route to this class of marine natural products and (2) to synthesize an aplysiatoxin analog(s) that should provide the crucial information for the three-dimensional model-building exercise. Synthetic, unnatural 3-deoxy-debromoaplysiatoxin (50c) played the indispensable role to suggest the three-dimensional model $\mathbf{5 1}$ for PKC activation by DAGs.

The synthesis of semi-synthetic photoprotein aequorin is the second example in this area. The photoprotein aequorin, isolated from jellyfish, is widely used as a sensitive intracellular probe for $\mathrm{Ca}^{2+}$; aequorin emits light in the presence of a trace amount of $\mathrm{Ca}^{2+}$, 
decomposing into apoaequorin, coelenteramide and $\mathrm{CO}_{2}$. Aequorin can be regenerated by incubation of apoaequorin with coelenterazine (9) in the presence of $\mathrm{O}_{2}$. Using this transformation, semi-synthetic aequorins were synthesized from synthetic coelenteramide analogs and recombinant apoaequorins. Interestingly, the semi-synthetic aequorins exhibited a wide range of the $\mathrm{Ca}^{2+}$-sensitivity, depending on the coelenterazine analogs used.

The synthesis of enterobactin analogs is the third example in this area. Enterobactin is a naturally occurring, physiolically active macrocyclic iron-sequestering agent. With the use of scyllo-inositol mono-orthoester as the platform, the chiral enterobactin analogs $R$ - and $S$-52a were synthesized and shown to form exclusively either the right-handed or the left-handed Fe(III)-complex. The stability constant of the ferric complex of 52a was estimated to be only slightly lower than that of enterobactin itself. It was shown that the lipophilicity/hydrophilicity of ferric complexes could be tuned with modification of the orthoester moiety present, cf., $\mathbf{5 2 b}$.

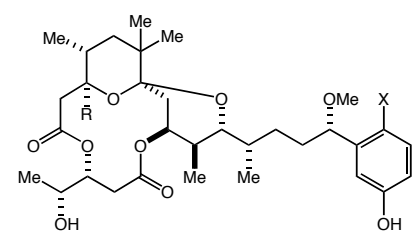

50a: Aplysiatoxin $(\mathrm{R}=\mathrm{OH} ; \mathrm{X}=\mathrm{Br})$ 50b: Debromoaplysiatoxin $(\mathrm{R}=\mathrm{OH} ; \mathrm{X}=\mathrm{H})$ 50c: 3-Deoxy-debromoaplysiatoxin $(R=H ; X=H)$

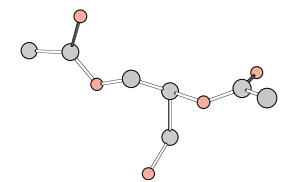

51: Diglyceride-template derived from aplysiatoxins

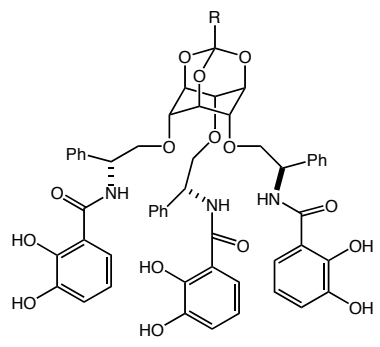

52a: scyllo-Inositol based enterobactin analog $(\mathrm{R}=\mathrm{H})$ 52b: scyllo-Inositol based enterobactin analog $\left(\mathrm{R}=n-\mathrm{C}_{10} \mathrm{H}_{21}\right)$

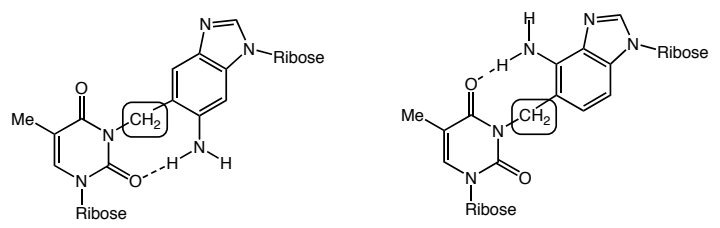

53a,b: Covalently cross-linked Watson-Crick base pair models

The $\mathrm{CH}_{2}$-bridged $C$-glycoside chemistry led to one additional twist - a covalently cross-linked Watson-Crick base-pair model. The $\mathrm{CH}_{2}$-bridged base-pair models may be uniquely suited to the chemical exploration of covalently cross-linked nucleosides/nucleotides. In addition to added chemical stability, these base-pair models should adopt only Watson-Crick or reverse Watson-Crick base-pairings while maintaining conformational flexibility along the $\mathrm{CH}_{2}$-bridge. Two types of base-pair models 53a,b were synthesized and then incorporated in anti-parallel and parallel DNA- 
oligomers. CD and NMR spectroscopic studies demonstrated that these DNAoligomers bearing a covalently cross-linked Watson-Crick base-pair model beautifully mimic the conformational properties found in the corresponding native duplexes. With this foundation, chemical ligation was studied.

\section{Other Studies}

Our research activities have been focused on the total synthesis of complex natural products. In order to carry out a synthetic work, it is crucially important to have mechanistic insights on synthetic reactions. Thus, from necessity, our group was engaged with the mechanistic studies on various synthetic reactions. The information gained was usually reported along with the total syntheses. However, one particular case is worthwhile to note. In planning a biomimetic synthesis of the aranotin/epicorazine-class of natural products, a possibility was recognized that the aranotin- and epicorazineskeletons might be formed via Baeyer-Villiger oxidation/Criegee rearrangement. With this background, a mechanistic study was conducted, thereby providing the compelling experimental evidence to suggest that the primary stereoelectronic effect governs BaeyerVilliger oxidation/Criegee rearrangement. With this mechanistic insight, it was shown that the hydroperoxides 54a and 55a selectively gave the aranotin-type and epicorazinetype products $\mathbf{5 4 b}$ and $\mathbf{5 5 b}$, respectively.
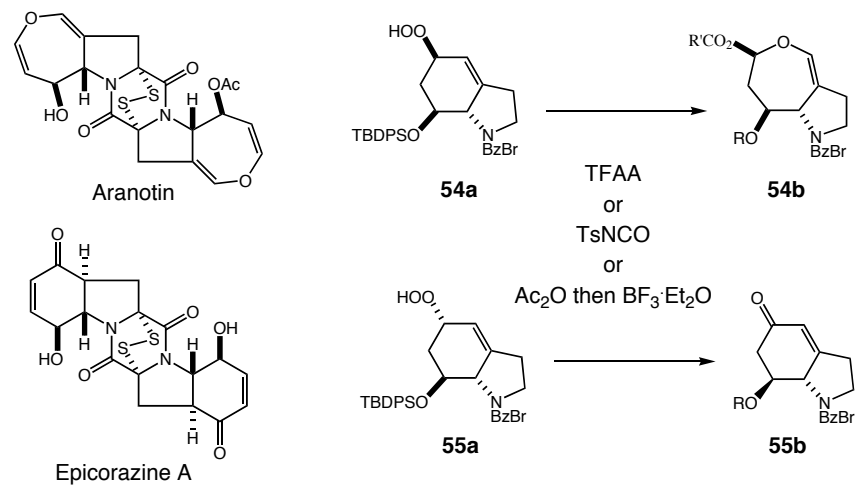

From time to time, we had a pleasure of running into unexpected, surprising chemical phenomena by chance. Some of them led us to conduct further studies on the observed phenomena, and one of them was the light emitting reaction of $(E)-2-$ benzenesulfonyl-3-phenyloxaziridine (56a) with strong bases.

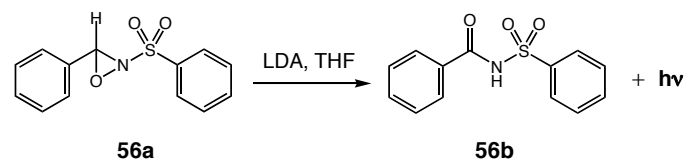

The proposed or presumed biosynthetic pathway often exerted a significant influence on our synthetic plan. In this connection, dinoflagellate and krill luciferins 4 and $\mathbf{5}$ were interesting; their structural similarity with chlorophylls $a$ and $b$ suggested that they are derived through an oxidative ring-cleavage at the $\mathrm{C} 1-\mathrm{C} 20$ bond of chlorophylls with retention of the $\mathrm{C} 20$ carbon as a carboxylate at ring D. Curiously, however, the 
chlorophyll catabolism involving the $\mathrm{C} 1-\mathrm{C} 20$ bond fission was not known. With this background, a biomimetic chlorophyll catabolism was studied, resulting in the discovery of a remarkably selective transformation of $\mathbf{5 7 a}$ to $\mathbf{5 7 b}$.

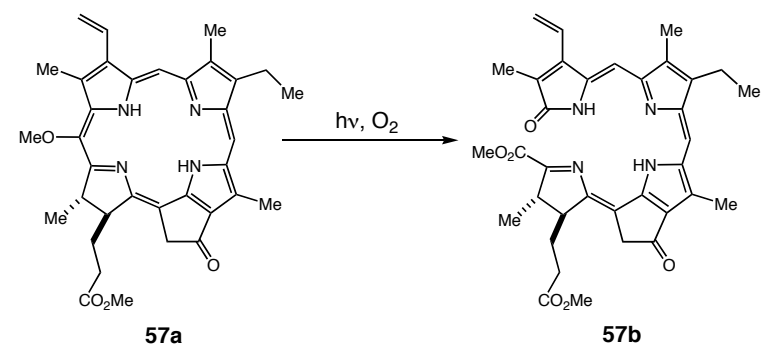

